\title{
Tuning the Structures and Properties of Porous Graphene in Laser-Induced Graphitization
}

\author{
Fangcheng Wang ${ }^{1,2,3}$, Kedian Wang ${ }^{1,2,3}$, Xia Dong ${ }^{* 1,2,3}$, Xuesong Mei ${ }^{1,2,3}$, Zhaoyang Zhai ${ }^{1,2,3}$, Jing Lv ${ }^{1,2,3}$, \\ Wenqiang Duan ${ }^{1,2,3}$ and Meng Gao ${ }^{1,2,3}$ \\ ${ }^{1}$ State Key Laboratory for Manufacturing System Engineering, Xi'an Jiaotong University \\ ${ }^{2}$ Shaanxi Key Laboratory of Intelligent Robots, Xi'an Jiaotong University \\ ${ }^{3}$ School of Mechanical Engineering, Xi'an Jiaotong University \\ E-mail: xdongxjtu@163.com
}

\begin{abstract}
Although graphene has excellent properties, it does not mean that all graphene-based devices will have high performance. Even though three-dimensional (3D) porous graphene are believed to be of great importance in many applications, the control of the microstructures and properties of this material to meet different application requirements in industrial practice remains challenging. In this report, we proposed a facile approach to tune the mophology and electrical conductivity of porous graphene during the laser-induced polyimide (PI) graphitization process. This porous graphene with mesoporous and macroporous has a large specific surface area, extremely low densities and good conductivity. Our results demonstrate a practical method to adjust structures and properties, and further benefit the various applications of porous graphene. This research also provides a promising candidate for porous, high performance and low-cost materials in the effective use of energy storage systems.
\end{abstract}

DOI: 10.2961/jlmn.2017.02.0020

Keywords: porous graphene; polyimide; laser; graphitization; regulation

\section{Introduction}

Graphene is a two-dimensional (2D) nano-sized carbon with excellent mechanical, thermal and electrical properties, which has good application prospect in energy storage field. To fully utilize and further explore the new functions of graphene, many researchers have made great efforts in the preparation of 3D porous graphene. They not only possess the intrinsic properties of $2 \mathrm{D}$ graphene sheets, but also provide excellent structures with high porosity and surface area. These features enable porous graphene materials to serve as key components in high-performance energy storage devices with their highly open porous structures allowing electrolytes access to the surface of porous frameworks [1, 2]. Despite its promise, there are still important fabrication challenges associated with synthesis and printing of 3D porous graphene [3-5]. Recently, much attention has been paid to the direct synthesis of porous materials using laser-induced graphitization on the PI sheets $[6,7]$. However, it remains unknown how to regulate the microstucture of the porous graphene during the laser induced graphitization process. Therefore, the aim of this study is to investigate the evolution of structures and properties of laser-induced graphene. Recently, our group developed a simple and scalable method to prepare hierarchical porous graphene films with defects from PI sheets using a high repetition rate nanosecond fiber laser in air [8]. We have demonstrated that the PI sheet can be graphitized by a high repetition rate nanosecond laser due to the heat accumulation effect. The process is new and has not been studied by various groups. By this approach, the polymeric sheet was chemically transformed into a honeycomb porous structure on graphitized film that composed of few-layer graphene or graphene stacks. In this report, we attempted to control the network structures and properties of 3D porous graphene by changing the laser
power.The optimized porous graphene has higher porosity and specific surface area, with pore sizes ranging from several nanometers to several tens of micrometers, and remarkable electrical conductivity. The simple process allows large-scale production at a low cost. This honeycomb porous graphene and its related functional materials have great application prospect in energy storage, catalyst carrier, sensor, water treatment and so on.

\section{Experimental}

\subsection{Fabrication of porous graphene}

Fig. 1 shows the laser scribing process. A laser scanning galvanometer system (CTI EC1000) with a JENar 170-1030 F-theta lens and equipped with a high repetition nanosecond fiber laser was used for irradiating PI sheets in air to generate porous graphene films. The single-mode nanosecond fiber laser characteristics include a wavelength of $1064 \mathrm{~nm}$, pulse duration of $10 \mathrm{~ns}$ and repetition rate of $800 \mathrm{KHz}$. The beam diameter of the nanosecond laser is $\sim 80 \mu \mathrm{m}$. The Kapton PI sheet with thickness of $100 \mu \mathrm{m}$ comes from commercial PI tape. Before exposure to laser irradiation, the PI sheets were cleaned in a solution of absolute alcohol and deionized water by ultrasonic cleaning for $10 \mathrm{~min}$, then rinsed in deionized water and dry naturally. In the laser irradiation process, the laser beam rastered over the PI surface to write patterns created by a graphic design software. For laser parameters in these experiments, line scanning speed, beam spot diameter and scanning space were fixed at $120 \mathrm{~mm} \mathrm{~s}^{-1}, 80 \mu \mathrm{m}$ and $16 \mu \mathrm{m}$, respectively. A gradually increasing laser output power from $3 \mathrm{~W}$ to $6 \mathrm{~W}$ was used to irradiate the PI sheet with a "Z" shape scanning path (The periodic structure of the scanning trajectory is shown in Fig. 1). The entire laser-induced graphitization process was performed under ambient conditions. 


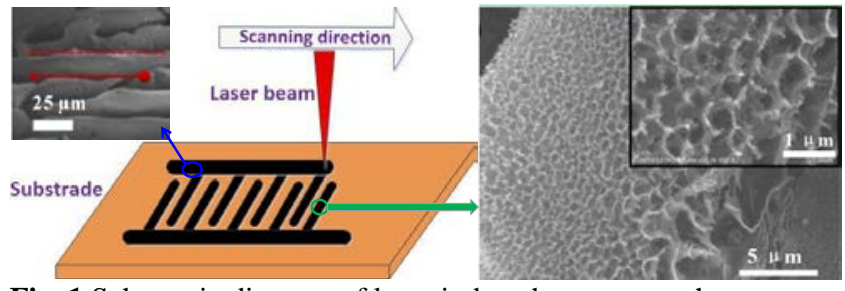

Fig. 1 Schematic diagram of laser induced porous graphene.

\subsection{Characterization and testing of graphene film}

Scanning electron microscopy (SEM) imaging was performed with Hitachi su-8010 at $10 \mathrm{kV}$ to examine the morphologies of the graphitic film features generated by nanosecond laser irradiation conditions. The Raman scattering spectra of modified area were collected by a horiba hr800 Raman microscope with a $633 \mathrm{~nm}$ excitation laser at a power of $17 \mathrm{~mW}$. Transmission electron microscope and high-resolution transmission electron microscopy (TEM/HR-TEM) images were carried out with JEOL JEM-2010 and JEOL JEM-2100F microscopes. The porous graphene flakes were scraped by the blade and sonicated in ethanol before being transferred onto a TEM grid. The square resistance were measured using an Everbing four-point probe meter. High-resolution atomic force microscope (AFM, Veeco-di Innova Model-2010USA) was used for testing morphological features and topological map of porous graphene flakes on silicon wafer. The surface area of HPG was measured with a Micromeritics Brunauer-Emmmett-Teller BET surface analyzer.

\section{Results and discussion}

The evolutions of the structure, morphology and properties of the features of laser induced graphene irradiated at different laser power with fixed gap distance $=16 \mu \mathrm{m}$ and speed $=120 \mathrm{~mm} \mathrm{~s}^{-1}$ were further characterized by SEM, TEM and Raman spectroscopy. As shown in Fig. 2(a-f), with the increase of the laser power, the ablation degree on the surface of the PI was gradually deepened, while the roughness decreases first and then increases. High magnification SEM images were acquired to examine the microscopic structures of the region irradiated under different laser writing conditions. The irradiated surface of PI presents a gauze-like porous structure at the lowest laser power of $3.0 \mathrm{~W}$. When the laser power varies in the range of 3.6-4.8 $\mathrm{W}$, honeycomb porous structures have been formed in the irradiated surface of PI that can effectively reduce the stacking of graphene sheets. Here, porous graphene films can be obtained with a laser power of 3.6 W, denoted as PG-3.6 W. Compared with conventional porous carbon material, the $3 \mathrm{D}$ hierarchical mesoporous and macroporous (See Fig. 3) graphene formed by the removal of internal non-carbon elements has a unique porous honeycomb system, which has mutually penetrating pores and the pore walls are made of graphene sheets. As the laser power increases to $5.4 \mathrm{~W}$, a layer of sediment particles of carbon attached to the surface of PI. Thus, when the power is less than $4.8 \mathrm{~W}$, the degree of graphitization of the PI increases as the laser power increases. As the laser power rises above $4.8 \mathrm{~W}$, partial oxidation starts to play an increasingly deleterious role in the quality of the films. This can also be further verified by the ratio of the D peak to the $G$ peak intensity in PG-5.4W
(Fig. 2g). It is well known that in the process of thermal and photo thermal pyrolysis, PI can produce various gases, such as $\mathrm{CO}_{2}$, $\mathrm{CO}$ and $\mathrm{NH}_{3}$ [9-11]. It is considered that the PI is pyrolyzed by laser heating in the one-step laser scribing process. As a consequence, the produced pyrolysis gases are quickly liberated to result in the "bombing" effect. Therefore the protruded porous structures with different shapes can be attributed to different laser power. The evolution of carbon microstructure upon different laser powers from $3.0 \mathrm{~W}$ to $6.0 \mathrm{~W}$ was confirmed by the Raman spectra as shown in Fig. 2g. The silent Raman features shown at $1333 \mathrm{~cm}^{-1}$ (D-band), $1590 \mathrm{~cm}^{-1}$ (G-band), and the 2D-band around $2660 \mathrm{~cm}^{-1}$ are consistent with the results that the film features of the graphitic structures created by $\mathrm{CO}_{2}$ laser irradiation of PI as few-layered graphenes or graphene stacks [12-15]. When the power $<4.2 \mathrm{~W}$, both the value of $I_{D} / I_{G}$ and $I_{G} / I_{2 D}$ decrease with the increase of laser power. When the power $>4.2 \mathrm{~W}$, both the value of $\mathrm{ID} / \mathrm{I}_{\mathrm{G}}$ and $\mathrm{I}_{\mathrm{G}} / \mathrm{I}_{2 \mathrm{D}}$ increase with the increase of laser power. As we know, the ratio of the peak intensity $\left(\mathrm{I}_{\mathrm{D}} / \mathrm{I}_{\mathrm{G}}\right)$ of the $\mathrm{D}$ and $G$ bands reflects the degree of defects in graphene, the ratio of the peak intensity $\left(\mathrm{I}_{2 \mathrm{D}} / \mathrm{I}_{\mathrm{G}}\right)$ of the $2 \mathrm{D}$ and $\mathrm{G}$ bands reflects the number of graphene layers. In the work reported here, we attempted to control the morphology and pore sizes distribution of porous graphene by changing the laser power during laser induced graphitization. Moreover, the electrical properties of the porous graphene is closely dependent on the pore size and degree of graphitization of PI. Fig. 2h shows the relationship between the conductivity and the laser power of graphene films. Therefore, the conductivity of this material can be adjusted within a certain range to meet the different functional requirements of the devices. Based on the unique multifunctional material, many applications in electronic and photonic devices, energy storage are possible to be achieved with the further developments.
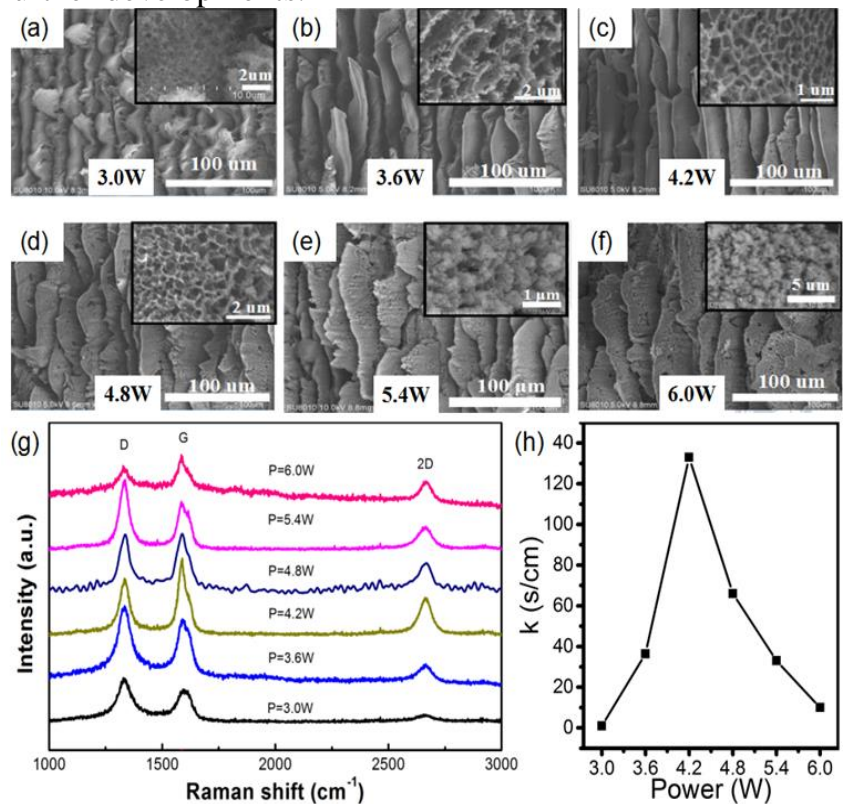

Fig. 2 SEM images of porous graphene obtained with different laser power ranging from $3.0 \mathrm{~W}$ to $6.0 \mathrm{~W}$ in increments of $0.6 \mathrm{~W}$. (a-f) The macroscopic morphology of porous graphene films at different power in air. Inset is the corresponding higher magnification SEM image. (g) Raman spectra of porouse graphene films under different laser power. (h) Correlations of the electric conductivities of HPG films with laser powers. 
Porous graphene films obtained with different laser power were further characterized with TEM and AFM. Fig. 3 shows that the micro morphology, the isotherms and pore size distribution of porous graphene flakes obtained under different laser power with a constant scanning speed $\left(120 \mathrm{~mm} \mathrm{~s}^{-1}\right)$ in air. As shown in Fig. 3(a-c), one can clearly see the laser irradiation generated graphene flakes with hierarchical macropores and mesopores on the surface of the graphene flakes. The results show that it provides an excellent ion and electron transport channel for the electrode materials of electrochemical energy storage devices [16]. When the laser power was $3.6 \mathrm{~W}$, the surface feature of graphene flakes present a coral-like structure with a diameter of 20-40 nm, which may be attributed to the curl of graphene nanosheets. As the laser power increases to $4.2 \mathrm{~W}$, the surface feature of graphene flakes show a distinctive root-like network structure. With further increasing the laser power, the structure of the root-like network gradually expanded. Fig. 3(d-f) are HR-TEM images of porous graphene nanosheets under the corresponding laser power. The clarity of the lattice fringe reflects the degree of graphitization of the PG-4.2w is best. As we all know, the AFM height image can reveal the thickness and size of the graphene flakes. Fig. 3(g-h) showed that the thickness of PG-4.2W flakes are predominantly 3 to $7 \mathrm{~nm}$, corresponding to the thickness of 5-10 layers graphene. Therefore, multilayer graphene or graphene stacks can be produced by laser ablated PI sheets. The BET specific surface area and porous structure characteristics of the PG-4.2W were investigated by nitrogen isothermal adsorption. As shown in Fig. 3(i), PG$4.2 \mathrm{~W}$ samples showed type IV isothermal adsorption desorption curve characteristics and $0.5<\mathrm{P} / \mathrm{P}_{0}<1.0$ showed H3 obvious hysteresis loop, the results show that the pore structure of mesoporous samples (2 50nm) [17]. The PG$4.2 \mathrm{~W}$ has a surface area of $\sim 70 \mathrm{~m}^{2} \cdot \mathrm{g}^{-1}$, with pore sizes $<$ $15 \mathrm{~nm}$. Pore sizes are mainly distributed at $3.29 \mathrm{~nm}, 7.35$ $\mathrm{nm}, 11.38 \mathrm{~nm}$ and $14.49 \mathrm{~nm}$.
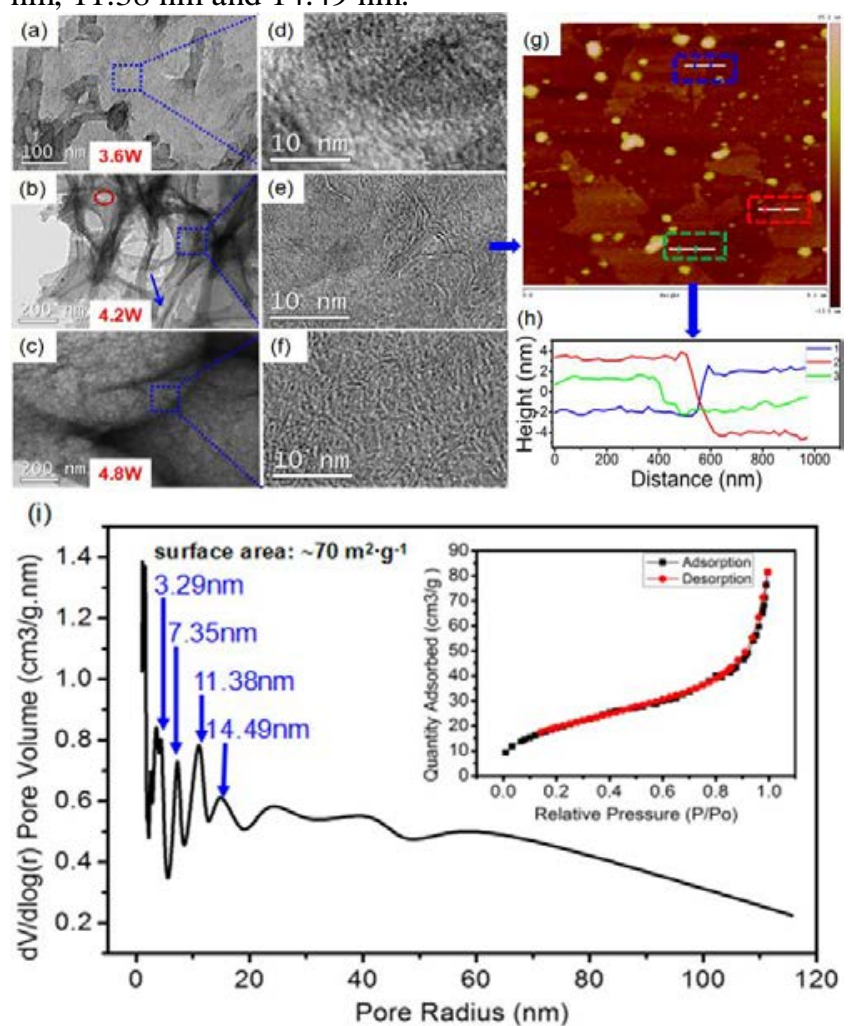

Fig. 3 (a-c) TEM images of porous graphene films obtained with different laser power in air. (d-f) HR-TEM images of porous graphene nanosheets under the corresponding power. The TEM image of the red-circled region and the blue arrow show the macroporous structures and mesoporous structure, respectively. (g-h) AFM height images of PG-4.2W nanosheets along with the height profile. (i) Pore size distribution of PG-4.2W tested by means of BET. Inset is $\mathrm{N}_{2}$ adsorption/desorption isotherms.

\section{Conclusion}

In summary, we have developed a one-step and scalable approach for the preparation of various porous graphene from PI sheets using high repetition rate laser irradiation in ambient conditions. The effect of nanosecond laser irradiation conditions on the morphology and property of the formed porous graphene features were studied by SEM, TEM and Raman spectra. The morphology and properties of 3D porous graphene can be controlled by laser power, which has many potential advantages in the field of energy storage. The main conclusions are as follows:

(1) The surface of the PI sheet irradiated by the laser beam in the air can form a 3D porous structure which is cross-linked to each other and synchronously realize the conversion to graphene. This porous structure can effectively reduce the stack of graphene sheets.

(2) The structural morphology and properties of PG can be controlled by changing the laser power which has a strong impact on the degree of graphitization.

(3) Compared with conventional porous carbon material, the 3D hierarchical mesoporous and macroporous graphene formed by the removal of internal non-carbon elements has a unique porous honeycomb system, which has mutually penetrating pores and the pore walls are made of graphene sheets.

As a candidate material for electrochemical energy storage devices, the $3 \mathrm{D}$ porous graphene system promise to provide excellent ion and electron transport network. We believe that the versatility of laser-induced technique demonstrated in this work can be highly valuable in fields ranging from energy storage and conversion to electronics.

\section{Declaration of conflicting interests}

The authors declare that there is no conflict of interest.

\section{Acknowledgments and Appendixes}

The authors would like to thank the National Natural Science Foundation of China (Grantno.51375374), Program for ChangJiang Scholars and Innovative Research Team in University (Grant No. IRT_15R54) for supporting this work.

\section{References}

[1] J. Hu, Z. Kang, F. Li, X. Huang: Carbon, 67, (2014) 221.

[2] J. X. Zhu, D. Yang, Z. Y. Yin, Q.Y. Yan, H. Zhang: Small, 10, (2014) 3480.

[3] K. H. Wu, H. H. Cheng, A. A. Mohammad, I. Blakey, K. Jack, I. R. Gentle, D. W. Wang: Carbon, 95, (2015) 738.

[4] Z. S. Wu, K. Parvez, X. L. Feng, K. Mullen: Nat. Commun., 4, (2013) 2487.

[5] T. Y. Kim, G. Jung, S. Yoo, K. S. Suh, R. S. Ruoff: ACS Nano., 7, (2013) 6899. 
[6] J. Lin, Z. W. Peng, Y. Y. Liu, F. Ruiz-Zepeda, R. Q. Ye, E. L.G. Samuel, M. J. Yacaman, B. I. Yakobson, J. M. Tour: Nat. Commun. , 5, (2014) 5714.

[7] L. Li , J. B. Zhang , Z. W. Peng , Y. L. Li , C. T. Gao , Y. S. Ji , R. Q. Ye ,N. D. Kim , Q. F. Zhong, Y. Yang, H. L. Fei, G. D. Ruan, J. M. Tour: Adv. Mater., 28, (2016) 838.

[8] F. C. Wang, K. D. Wang, X. Dong, X. S. Mei, Z. Y. Zhai, B. X. Zheng, J. Lv, W. Q. Duan, W. J. Wang: Appl. Surf. Sci. , 419, (2017) 893.

[9] M. Inagaki, N. Ohta, Y. Hishiyama: Carbon, 61, (2013) 1.

[10] G. H. Wynn, A. W. Fountain: J. Electrochem. Soc., 144, (1997) 3769.

[11] J. H. Brannon, J.R. Lankard: Appl. Phys. Lett., 48, (1986) 1226.
[12] J. Lin, Z. Peng, Y. Liu, F. Ruiz-Zepeda, R. Ye, E.L.G. Samuel, M. J. Yacaman, B. I. Yakobson, J. M. Tour: Nat. Commun., 5, (2014) 5714.

[13] A. C. Ferrari, J.C. Meyer, V. Scardaci, C. Casiraghi, M. Lazzeri, F. Mauri, S. Piscanec1, D. Jiang, K. S. Novoselov, S. Roth, A. K. Geim: Phys. Rev. Lett., 97, (2006) 187401.

[14] A. C. Ferrari: Solid State Commun., 143, (2007) 47.

[15] A.C. Ferrari, D.M. Basko: Nat. Nanotechnol., 8, (2013) 235.

[16] Y. W. Zhu, S. Murali, M. D. Stoller, K. J. Ganesh, W. W. Cai, P. J. Ferreira, A. Pirkle, R. M. Wallace, K. A. Cychosz, M. Thommes, D. Su, E. A. Stach, R. S. Ruoff: Science, 332, (2011) 1537.

[17] X. Tang, Z. H. Liu, C. Zhang, Z. Yang, Z. Wang: J. Power Sources, 193, (2009) 939.

(Received: February 23, 2017, Accepted: September 14, 2017) 